\title{
Perfil sensorial del Clon de cacao (Theobroma cacao L.) CCN51 (primera cosecha de 2015)
}

\author{
Clone sensory profile of cocoa (Theobroma cacao L.) CCN51 (first vintage 2015)
}

\author{
Quintana F. Lucas, Gómez C. Salomón, García J. Alberto, Martínez Nubia \\ Universidad Nacional Abierta y a distancia UNAD, Escuela de ciencias Básicas, tecnología e ingeniería, \\ Programa de ingeniería de alimentos, Grupo de Investigación GIA UNAD. Carrera 27\#40-43 \\ Bucaramanga. \\ Federación nacional de cacaoteros de Colombia. FEDECACAO
}

Recibido 26 de Abril 2015; aceptado 18 de Mayo 2015

\section{RESUMEN}

En este trabajo se realizó la evaluación preliminar en la primera cosecha del 2015 del clon de cacao CCN 51 procedente de los cuatro sistemas agroforestales en que se produce en Colombia: La "montaña Santandereana" con muestras de San Vicente de Chucuri, (S); "Valle interandino Seco" con muestras de Gigante (H); "Bosque Húmedo tropical" con muestras de Arauca y "zona media baja Cafetera" con muestras de San Jerónimo, (Ant.). Obtenido el cacao seco se procedió en el laboratorio a preparar los licores de cacao y a someterlos a evaluación sensorial por parte del grupo de jueces expertos del laboratorio de Fedecacao ubicado en San Vicente de Chucuri a los cuales se le han realizado pruebas de validación para garantizar un grado de confiabilidad del 99.5\%. El perfil de sabor se estableció de acuerdo a la Norma técnica colombiana NTC 3929, utilizando la técnica de perfil de sabor por consenso establecida en esta misma y generar los respectivos perfiles para cada muestra y un perfil global representativo de los cuatro sistemas en estudio. Se estableció un perfil por sistema y consolidar uno global para el clon CCN 51, el cual, es consistente con perfiles realizados en anteriores estudios en donde se resalta su sabor a cacao y algunas notas de sabores especiales como son nuez y dulce.

Palabras clave: Clon CCN51, evaluación sensorial, fermentación, perfil sensorial, sistemas agroforestales.

\section{ABSTRACT}

*Autor a quien debe dirigirse la correspondencia. E-mail: lucas.quintana@unad.edu.co
In this work we made the preliminary evaluation of the first harvest of the 2015 of the clone of cocoa CCN51 from the four agroforestry systems in which is produced in Colombia: The Santander Mountains with samples of San Vicente del Chucuri, (S); the interandean dry valley with samples of Gigante $(H)$; wet tropical forest with samples of Arauca and the low medium coffee zone with samples of San Jerónimo (Ant). 
Obtaining the dry cocoa we procedure on the laboratory to prepare the cocoa liquors to submit it to a sensory evaluation by a group of experts of the Fedecacao laboratory located in San Vicente del Chucuri, those experts have made validation tests to guarantee a reliability of the $99,5 \%$. The flavor profile was established by the Colombian technical norm NTC 3929, using the technique of the flavor profile accorded in this one to generate the respective profiles for each sample and a representative global profile of the four studied systems. Achieving obtain a profile per system and consolidate a global one for the clone CCN 51 which is consistent with the made profiles in previous studies where they stand out its cocoa flavor and some notes of special flavors like nuts and sweet.

Keywords: clone $\operatorname{CCN} 51$, sensory evaluation, fermentation agroforestry systems.

\section{INTRODUCCIÓN}

El Clon de cacao CCN 51 (Colección Castro Naranjal) es originario de Ecuador en donde fue obtenido de cepas de cacao de Iquitos, Criollo y amelonado por Homero Castro en 1965, es un clon resistente a diferentes plagas que lo han hecho un cacao de alta productividad y resistencia, en Ecuador ha incrementado la producción de 20.000 toneladas a anuales a 100.000 toneladas anuales entre el año 2005 a 2013. Este clon ha sido también adoptado en países como Perú y Colombia en donde ha tenido alta promoción y desarrollo debido a su alta productividad que oscila entre 2000 a 3000 toneladas por hectárea en condiciones óptimas de producción (EI Comercio, 2015). Castro Naranjal, investigo la población de cacao del alto Amazonas (Ecuador), coleccionando material genético para usarlos en programas de cruzamiento con variedades Trinitarias y otros cultivares, buscando un clon de alta calidad y gran productividad resistente a las enfermedades "Escoba de Bruja", "Monilia" y Ceratocystis (Mal del Machete) (Crespo, 1997). En el mundo este clon ha sido catalogado como un cacao corriente por su alta presencia de acidez, astringencia y pocas notas de sabores florales, frutales y a nuez que presentan en mayor proporción los cacaos criollos y especiales.
En este trabajo se realizó la evaluación preliminar del clon de cacao CCN 51 procedente de los cuatro sistemas agroforestales en que se produce en Colombia: La "montaña Santandereana" con muestras de San Vicente de Chucuri, (S); "Valle interandino Seco" con muestras de Gigante $(H)$; "Bosque Húmedo tropical" con muestras de Arauca y "zona media baja Cafetera" con muestras de San Jerónimo, (Ant.). Se recolectaron muestras de la primera cosecha del 2015, meses de Mayo, Junio y Julio y proyectando una segunda toma de muestras para la segunda cosecha del 2015. El manejo y trasformación de las muestras (cacao en baba) se realizó en la granja "Villa Mónica" de la federación colombiana de cacaoteros Fedecacao en San Vicente de Chucuri, garantizando el cumplimiento del protocolo de fermentación establecido en su guía técnica para el agricultor, en este protocolo se establece el proceso de fermentación y secado (Fedecacao, 2013). Una vez obtenido el cacao seco se procede en el laboratorio a preparar los licores de cacao y a someterlos a evaluación sensorial por parte del grupo de jueces expertos del laboratorio de Fedecacao a los cuales se le han realizado pruebas de validación para garantizar un grado de confiabilidad del 99.5\% ( NTC 4129, Sukha, 2007). 
El perfil de sabor se estableció de acuerdo a la Norma técnica colombiana NTC 3929, utilizando la técnica de perfil de sabor por consenso establecida en esta misma y así se pudo generar los respectivos perfiles para cada muestra y un perfil global para el país (NTC 3929, NTC 4934, Ramos, 2014).
Se confirman algunos de los referentes que se tienen sobre la influencia del grado de fermentación en la calidad final del licor en la presencia de algunos sabores como son el ácido, el astringente y la presencia de sabores buenos como sabor dulce, nuez, floral o frutal.

\section{MATERIALES Y MÉTODOS}

El presente trabajo correspondió a un estudio descriptivo del perfil sensorial del licor de cacao obtenido del clon de cacao CCN 51 de amplia utilización en la actualidad en nuestro país por sus condiciones de resistencia enfermedades y alta productividad.

\section{Toma de muestras}

Se consideró la toma de muestras en la primera cosecha del 2015 y en cuatro regiones de Colombia en donde cada una representa uno de los cuatro sistemas agroforestales de producción de cacao y que se conocen como se mencionó anteriormente. Las localidades seleccionadas son: La Granja Villa Mónica, de la Vereda Mérida en el municipio de San Vicente de Chucurí, de propiedad de la Federación Nacional de Cacaoteros-FEDECACAO, a 3 kilómetros del casco urbano del municipio, a una altura de 879 m.s.n.m. con una temperatura promedio de $24^{\circ} \mathrm{C}$, con una extensión de 4.5168 has representa la montaña Santandereana. La Granja Alto Magdalena tiene una extensión de 3.5 hectáreas y una altura sobre el nivel del mar de 975 metros, presenta un régimen bimodal de lluvias, y la precipitación promedia en la zona es de 1100 milímetros, y una temperatura promedio de $25^{\circ} \mathrm{C}$. Las plantaciones de cacao en la granja Santa Elena en los últimos 5 años se han venido renovando por medio de siembra Nueva, correspondiente al valle interandino seco. La Granja Rafael Rivera se encuentra ubicada al occidente del departamento de Antioquia, en el municipio de San Jerónimo, a un kilómetro por la antigua vía a Medellín. Posee una extensión de 4.0 hectáreas, se encuentra a una altura de 750 metros sobre el nivel del mar, con una temperatura media de 28 grados centígrados, una humedad relativa de $80 \%$ y una precipitación aproximada de 1100 milímetros distribuidos en el año, esta granja representa la zona media baja cafetera. La Granja Santa Elena propiedad de Fedecacao se encuentra en la vereda Campo Alegre, en el municipio de Arauquita, con un área en cacao de 6 hectáreas con cultivos jóvenes entre los 4 y 18 años, representa el bosque húmedo tropical.

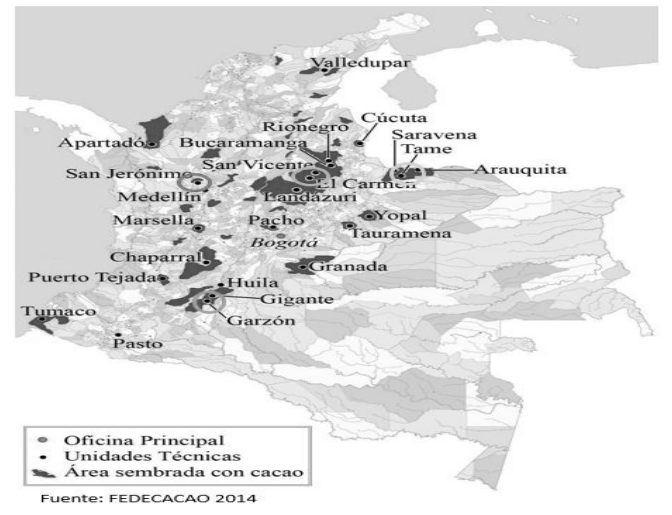

Figura 1. Mapa Cacaotero colombiano

En el mapa anterior se puede ver la ubicación de las granjas en las cuales fueron tomadas las muestras de cacao CCN 51. Una vez recolectadas las muestras correspondientes a cada región fueron procesadas las mazorcas y enviado en baba el cacao al Municipio de San Vicente de Chucuri, procediendo a fermentar en la granja de Villa Mónica según protocolo de fermentación, secado y tostado establecido por Fedecacao y elaborado licor de cacao en el laboratorio de Evaluación Sensorial de Fedecacao en San Vicente de Chucuri. 


\section{Evaluación licores de CCN 51 de las cuatro regiones}

Una vez obtenidos los licores de cacao se procedió a determinar el perfil de sabor de acuerdo a la GTC 165 y la NTC 3929 por consenso y establecer los perfiles de sabor correspondientes. Se presentaron las muestras debidamente codificadas y de manera que se eliminen los posibles errores, se determinó los sabores básicos correspondientes a acidez, astringencia, amargo, dulce. Sabores específicos a cacao, floral, frutal, nuez. Sabores adquiridos como verde, moho, picante y otros (Ramos 2014, Jiménez 2011, Sukha 2007). Para el registro de la evaluación se utilizó formato desarrollado para el registro de la intensidad de cada atributo en una escala de 0 a 10, la calidad del atributo en una escala de 0 a 10, la intensidad del regusto de o a 10 y la calidad global del licor de 0 a 10 (Appcacao, 2015). Inicialmente, el evaluador trabajó individualmente, registrando las características o notas de sabor. Tan pronto se tienen las notas de sabor, se recomienda consensuarlas. Con base en las notas definidas, se determina el orden de percepción, intensidad, sabor residual o persistencia, o ambos, y luego se evalúa la impresión total (NTC 3929).

Una vez obtenidos los resultados se procedió al cálculo del perfil por medio del promedio aritmético.

\section{RESULTADOS Y DISCUSIÓN}

El porcentaje de fermentación es un indicador clave que nos proyecta la calidad final sensorial del licor del cacao de cualquier clon, los parámetros de proceso como tiempo y temperatura son claves para el logro de una fermentación adecuada que permita el desarrollo de todos los sabores y aromas que caracterizan un buen cacao. En este proceso se obtienen valores intermedios de fermentación como se observa en la figura 2, que dan como resultado una buena fermentación de conformidad a las características del grano de cacao del clon CCN 51.

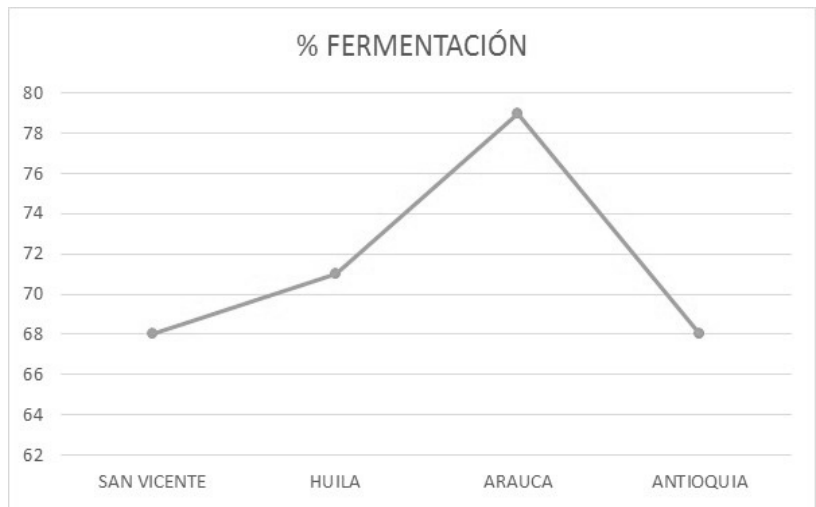

Figura 2. Porcentaje de fermentación
Posteriormente se procedió a evaluar cada licor, con la participación de 9 jueces expertos pertenecientes al panel de evaluación del laboratorio de Fedecacao, en San Vicente de Chucuri. En los resultados se observa una tendencia de los cuatro licores correspondientes a cada sistema agroforestal hacia los sabores básicos y algunos adquiridos como son el sabor a nuez, dulce y frutal (figura 3).

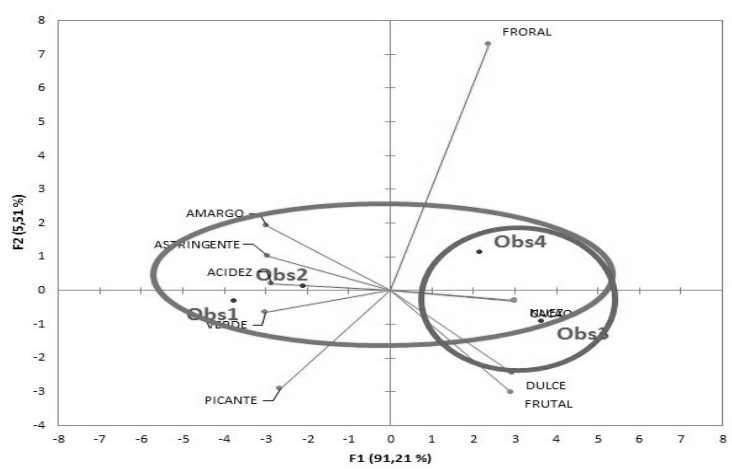

Figura 3. Análisis de componentes licores de CCN 51

En el perfil sensorial por región (figura 4), se puede establecer cuál de los cuatro licores presenta un mejor perfil, este perfil lo podemos relacionar con el porcentaje de fermentación y confirmar que un buen proceso genera un buen perfil, coincidiendo que el licor de la región de Arauca que tuvo un $78 \%$ 
de fermentación es el que presenta un menos sabor ácido, astringente y amargo, presenta un mayor sabor a cacao, notas de dulce, frutal y nuez. En la calificación global del licor fue considerado con un puntaje de 7 por parte de los jueces.

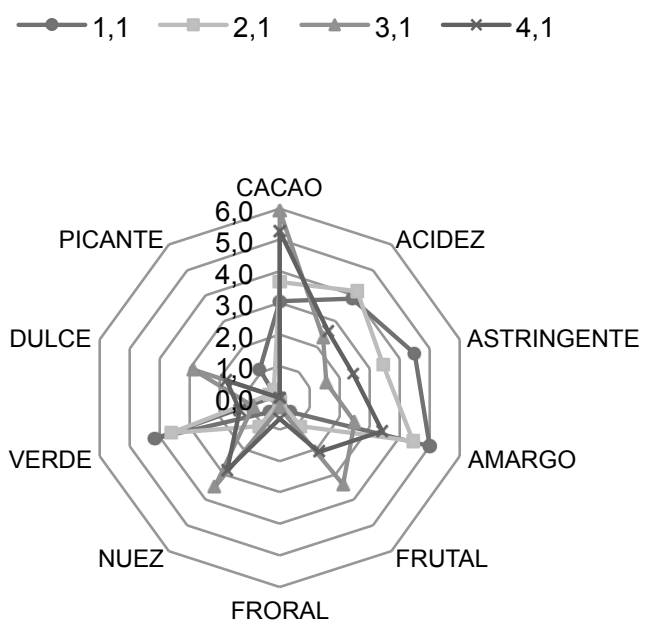

Figura 4. Perfil sensorial del CCN51 por región

El perfil global para este clon con los datos de esta cosecha primera del año 2015 , coincide con perfiles obtenidos en otras mediciones realizadas en el 2012 y 2013 por Quintana, L. (2013) por Perea et al., manteniéndose las características de cacao, acidez, astringencia, amargo, dulce, verde, frutal y nuez. Todos relacionados con el proceso de manejo pos cosecha en especial con el proceso de fermentación.

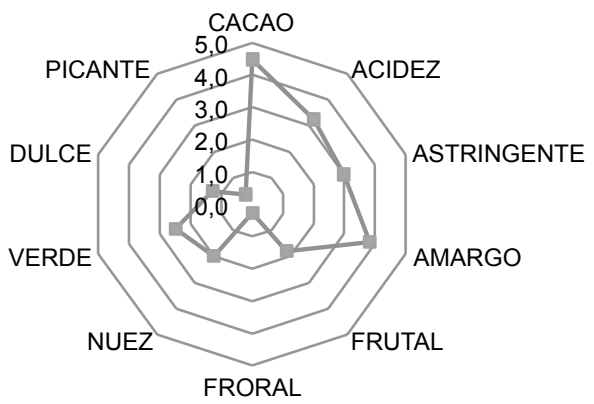

Figura 5. Perfil global CCN 51 primera cosecha 2015

Comparando el perfil del licor de cacao, primera cosecha de 2015 (figura 5), con el obtenido en el 2013 por Perea et al., (2013) y Jiménez (2011), se mantienen en proporción las características obtenidas por el panel de San Vicente. El índice global de la calidad del licor de cacao correspondiente a la evaluación de los sabores básicos, especiales y adquiridos generado por los miembros del panel fue de 4.0 sobre 10 para la muestra $1,4,5$ sobre 10 para la muestra 2, 6.9 para la muestra 3 y de 6.7 para la muestra 4. Esto permite establecer que la calidad del licor obtenido del clon CCN 51 es de un cacao de buena calidad que puede ser utilizado para la producción de chocolates amargos sin sabores especiales o en mezclas con otros licores de mejor calidad (APPCACAO, 2015).

\section{CONCLUSIONES}

El perfil sensorial obtenido para el clon CCN51 es consistente con el obtenido en otros países como Ecuador y en estudios realizados en los años 2012 y 2013 realizados por los autores y por Perea et al en el año 2013, ratificando su condición de cacao tipo forastero especial para la elaboración de chocolate de mesa. El nivel de fermentación es factor determinante en los resultados de los atributos finales de sabor a cacao, astringente, ácido, amargo, verde y floral.
Los resultados manifiestan una tendencia de los cuatro licores correspondientes a cada sistema agroforestal hacia los sabores básicos y algunos adquiridos como son el sabor a nuez, dulce y frutal. La cooperación entre la UNAD y la Federación Nacional de cacaoteros, Fedecacao ha permitido un avance importante en la investigación sensorial del cacao Colombiano con la finalidad de fortalecer el sector productivo y generar mejores condiciones de negociación para los productores. 


\section{REFERENCIAS BIBLIOGRÁFICAS}

Appcacao -USAID-TCHO-EQUAL. 2014. Manual de la Ficha de Catación de cacao. LIMA - PERU: APPCACAO.

Carpenter R. 2002. Análisis sensorial en el desarrollo y control de la calidad de alimentos. Zaragoza: Editorial Acribia, S.A.

Crespo E y Crespo F. 1997. Cultivo y Beneficio del cacao CCN51. Guayaquil - Ecuador: El Conejo.

El cacao CCN-51. 2015. El comercio, pp. http://www.elcomercio.com/actualidad/negocios/cacao-ccn-51paso-de.html.

Ibáñez F.C. y Barcina Y. 2001. Análisis Sensorial de Alimentos. Métodos y Aplicaciones. Barcelona: Springer-Verlag Ibérica.

ICONTEC. 2007. GTC 165, análisis sensorial. Metodología. Guía general. Bogotá, D.C.: Icontec.

ICONTEC. 1997. Guía general para la selección, entrenamiento y seguimiento de evaluadores. Parte 1. Evaluadores seleccionados. NTC 4129. Bogotá D.C.: ICONTEC.

ICONTEC. 2009. NTC 3929. Análisis sensorial. Metodología. Métodos del perfil del sabor. Bogotá D.C., Colombia: ICONTEC.

ICONTEC. 2001. NTC 4934. Análisis sensorial. Metodología. Guía general para establecer un perfil sensorial. Bogotá D.C., Colombia.: ICONTEC.

Jimenez J., Amores F., Nicklin C., Rodriguez D., et al., 2011. Micro fermentación y análisis sensorial para la selecciòn de àrboles superiores de cacao. Quevedo: INIAP.

Perea A., Martínez N., Aránzazu F., y Cadena T. 2013. Características de calidad del cacao e Colombia. Catálogo de 26 cultivares. (Primera Edición). Bucaramanga-Colombia: División de publicaciones UIS.

Quintana F. L. \& Gómez C. S. 2012. Evaluación sensorial del cacao producido en San Vicente de Chucurí (S). Universidad Nacional Abierta y a Distancia UNAD, Bucaramanga.
Quintana L. y Gómez S. 2013. Evaluación de la calidad sensorial del grano de cacao en tres pisos térmicos de la zona de San Vicente de Chucuri para los clones CCN51, ICS60 e ICS 95. Bucaramanga.: UNAD.

Ramos Gladys, González Néstor, Zambrano Alexis y Gómez Álvaro. 2013. Olores y sabores de cacaos (Theobroma cacao L.) venezolanos obtenidos usando un panel de catación entrenado. Revista científica UDO Agrícola, 13, 114-127.

Sancho J., E. Bota y J.J. de Castro. 1999. Introducción al Análisis Sensorial de los alimentos.

Stevenson C., Corven J. \& G. 1993. Manual para el análisis de cacao en el laboratorio. San José, Costa Rica: IICA.

Sukha D., Butler D., Umaharan P. y Boult E. 2007. The use of an optimised organoleptic assessment protocol describe ann quantify different flavour attributes of cocoa liquors made from Ghana and Trinitariio beans. Eur Food Res Techno, (SpringerVerlag), 1-9. 